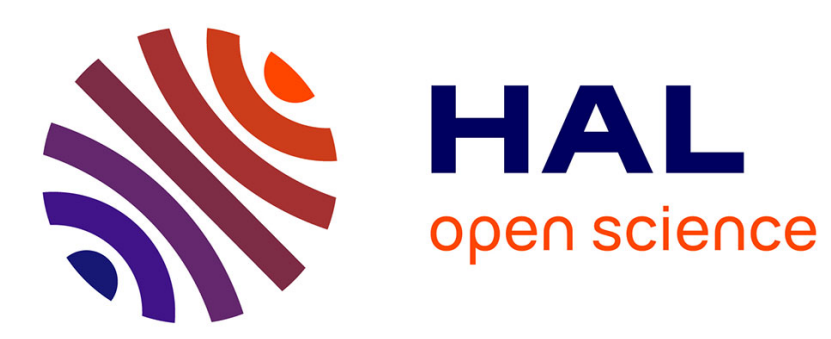

\title{
Growth of two dimensional domains in copolymer thin films
}

\author{
D. Ausserré, D. Chatenay, G. Coulon, B. Collin
}

\section{To cite this version:}

D. Ausserré, D. Chatenay, G. Coulon, B. Collin. Growth of two dimensional domains in copolymer thin films. Journal de Physique, 1990, 51 (22), pp.2571-2580. 10.1051/jphys:0199000510220257100 . jpa-00212554

\section{HAL Id: jpa-00212554 https://hal.science/jpa-00212554}

Submitted on 1 Jan 1990

HAL is a multi-disciplinary open access archive for the deposit and dissemination of scientific research documents, whether they are published or not. The documents may come from teaching and research institutions in France or abroad, or from public or private research centers.
L'archive ouverte pluridisciplinaire HAL, est destinée au dépôt et à la diffusion de documents scientifiques de niveau recherche, publiés ou non, émanant des établissements d'enseignement et de recherche français ou étrangers, des laboratoires publics ou privés. 
Classification

Physics Abstracts

64.00

\title{
Growth of two dimensional domains in copolymer thin films
}

\author{
D. Ausserré, D. Chatenay, G. Coulon and B. Collin
}

Equipe de Physique de la Matière Condensée, CNRS URA n 1379 and Université Pierre et Marie Curie, Institut Curie, Section de Physique et Chimie, 11 rue Pierre et Marie Curie, 75231 Paris Cedex 05, France

(Received 25 June 1990, accepted 20 July 1990)

\begin{abstract}
Résumé. - Lorsqu'on trempe des films très minces (1 $000 \AA$ d'épaisseur) de copolymères déposés sur un substrat solide, des domaines circulaires apparaissent en relief à la surface de l'échantillon. Ce phénomène est dû à la formation d'un ordre lamellaire dans le film qui s'accompagne d'une quantification de son épaisseur locale. Les domaines peuvent correspondre à des dépressions ou des élévations, selon l'épaisseur du film initial. Après avoir postulé que la structure des bords des domaines est une simple dislocation, on établit les équations qui gouvernent leur croissance. On montre en particulier que les écoulements de polymère qui permettent cette évolution doivent prendre place dans une bicouche incomplète enfermée entre deux monocouches complètes, et on fait l'hypothèse que la dissipation provient essentiellement du frottement entre couches. Le cas d'un trou unique dans un film de surface finie est finalement examiné en détail. Son rayon évolue comme la puissance $1 / 3$ de sa durée de vie.
\end{abstract}

\begin{abstract}
Quenching experiments on very thin films $(\simeq 1000 \AA)$ of symmetric diblock copolymer deposited on solid substrates result in the formation of circular relief domains at the free surface of the sample. It is due to the quantization of the possible thicknesses of the sample which accompanies the lamellar ordering of the two species parallel to the substrate. Depending on the average thickness of the film, the domains can be depressions or elevations. Based on the conjecture of a simple dislocation structure of the domain edge, equations governing the time evolution of the domain size distribution are proposed. The flows involved in the domain growth are shown to be confined into a bilayer which is disrupted at the dislocation lines. The hypothesis is made that interlayer frictions dominate. The growth kinetics of a single hole in a finite film is finally solved explicitely. Its radius is found to decay as a $1 / 3$ power law of its life time.
\end{abstract}

Diblock copolymers A-B in the liquid state undergoe an order-disorder transition at a temperature $T_{\mathrm{MST}}$, the so-called Microphase Separation Temperature [1]. The disordered state is often not observable in practice since $T_{\mathrm{MST}}$ can be out of the temperature range in which species A and B remain chemically stable. However disordered mixtures of A and B can be prepared in the solid state, i.e. at temperature $T$ below the glass temperatures $T_{\mathrm{g}_{\mathrm{A}}}$ and $T_{\mathrm{g}_{\mathrm{B}}}$ (we set $T_{\mathrm{g}_{\mathrm{A}}}<T_{\mathrm{g}_{\mathrm{B}}}$ ) of $\mathrm{A}$ and $\mathrm{B}$ polymers.

From there, it is possible to proceed to a "quenching " experiment by increasing $T$ from $T<T_{\mathrm{g}_{\mathrm{A}}}$ to $T$ such that $T_{\mathrm{g}_{\mathrm{B}}}<T<T_{\mathrm{MST}}$. Further evolution of the sample is the same as would 
be obtained when deeping it abruptly from an equilibrium disordered liquid state at $T>T_{\mathrm{MST}}$ into the ordered liquid state region $T_{\mathrm{g}_{\mathrm{B}}}<T<T_{\mathrm{MST}}$.

In the ordered equilibrium state, a layered structure is obtained when $A$ and $B$ chains have equal lengths. It corresponds to a sequence ... ABBAABBAABBAAB ... of $A$ and $B$ blocks [2]. The characteristic period of the lamellar stacking is the thickness of an ABBA sequence. $L$ results from a balance between the surface energy of the $A B$ interfaces and the elastic energy stored in elongated chains [3]. A typical value of $L$ is $400 \AA$ for a diblock copolymer of molecular mass 100000 [4].

Very thin films of copolymers can be made by spin coating at room temperature $T_{0}$. They are homogeneous in thickness, solid and macroscopically disordered [4]. Annealing at $T>T_{\mathrm{g}_{\mathrm{B}}}$ then results in an homogeneous liquid film which is ordered if $T<T_{\mathrm{MST}}$ (and probably also for $T \geq T_{\mathrm{MST}}$ ). Furthermore, the solid substrate is orienting the lamellae by selecting the component which leads to the lowest surface energy of the solid-polymer interface, say the A species for instance. Likewise, one component only is present at the free surface of the film, due to the difference in $A$ and $B$ surface energies $\gamma_{A}$ and $\gamma_{\mathrm{B}}$. We take $\gamma_{\mathrm{A}}>\gamma_{\mathrm{B}}$, so that $\mathrm{B}$ is selected. Within these hypothesis, the only possible sequences encountered in the ordered state are $\mathrm{AB}(\mathrm{BAAB})_{n}$. The associated thicknesses are $d_{n}=\left(n+\frac{1}{2}\right) L$, where $n$ is an integer.

The initial disordered film obtained by spin coating has a thickness $d$ which in general is such that $d_{n}<d<d_{n+1}$ for some $n$. After a sufficient annealing time $t$, the lamellar order has been completed and the local thickness of the film can be either $d_{n}$ or $d_{n+1}$, but not $d$. This results in the formation of coexisting regions of thicknesses $d_{n}$ and $d_{n+1}$ all over the surface. These domains can be observed using standard techniques of optical microscopy [5].

Figure 1 shows examples of such domains in two typical situations. These micrographs represent PS/PMMA copolymer films deposited on silicon substrates. We have $n=1$ in all cases. The pictures have been taken after annealing times of order three days at $T=170{ }^{\circ} \mathrm{C}$.

Figure 1a corresponds to $0<\frac{d-d_{1}}{L} \ll \frac{1}{2}$. Islands of thickness $d_{2}$ are emerging from a continuous background of thickness $d_{1}$.

Figure $1 \mathrm{~b}$ is obtained with $\frac{1}{2} \ll \frac{d-d_{1}}{L}<1$. Holes of depth $L$ form in a continuous film of thickness $d_{2}$.

These patterns are very similar to those encountered in first order transition kinetics [6], in far off critical situations.

Patterns resembling spinodal decomposition and critical situations have also been obtained and will be presented in a forthcoming paper. In all cases, the characteristic size of the pattern is growing with time. Quantitative kinetics studies are under progress, and detailed reports on these experiments will also be given elsewhere.

In situations illustrated in figure 1, domains are growing on the spot without appreciable diffusion. The growth mechanism appears to be twofold : large domains are growing at the expense of smaller ones, like in Ostwald ripening [7]; and growing neighbour domains sometimes recover each other and coalesce. In both cases, their circularity is either conserved or restored. It is indicative of the presence of a line tension at the domain edge. At the moment, we do not go further in reporting experimental observations, even qualitatively. Here, we just use the above-mentioned elements as a starting point for theoretical speculations upon the growth mechanism.

In a first section, a molecular model for the structure of the copolymer film will be 


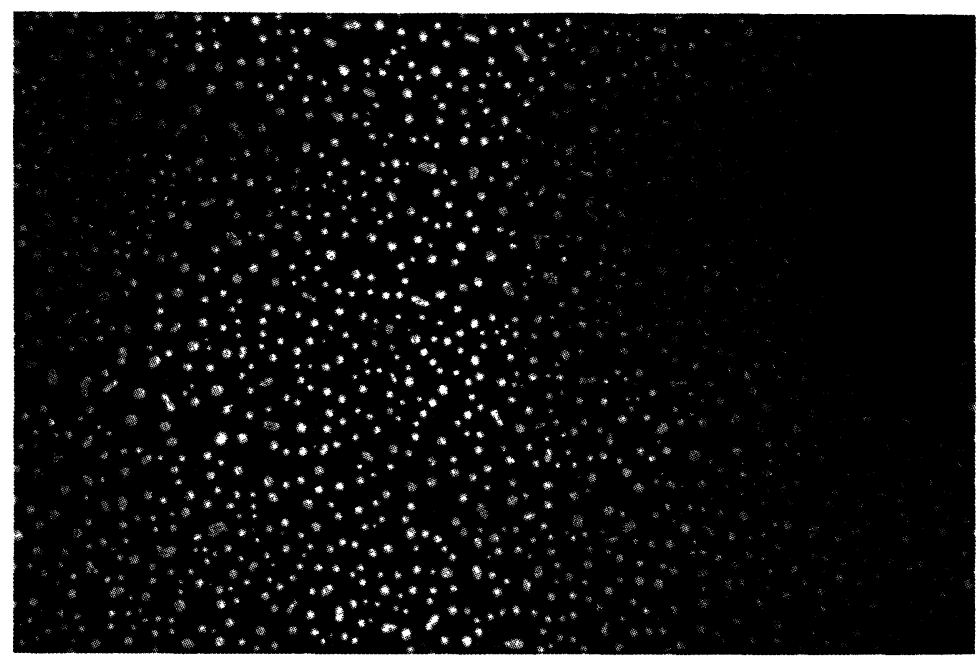

a)

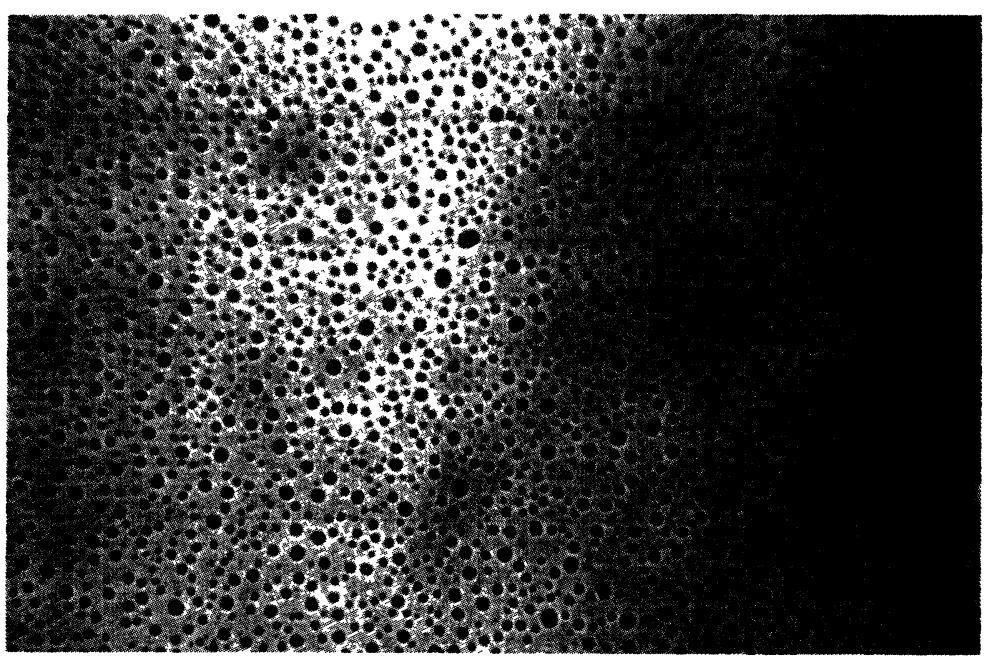

b)

Fig. 1. - a) Micrograph of relief domains at the free surface of a PS/PMMA diblock copolymer film deposited on a silicon wafer and annealed for 40 hours at $190{ }^{\circ} \mathrm{C}$. The molecular mass of the copolymer is 100000 . The circular domains are depressions of height $400 \AA$ in a continuous film of thickness $1200 \AA$. The picture has been obtained by white interferometry on a reflective optical microscope. The largest dimension of the picture is $300 \mu \mathrm{m}$. b) Ibidem with elevations of thickness $\simeq 400 \AA$ emerging from a continuous film of thickness $800 \AA$. Annealing time is 200 hours.

proposed, and qualitative differences between the situations $\frac{d-d_{n}}{L}<\frac{1}{2}$ and $\frac{d-d_{n}}{L}>\frac{1}{2}$ will be underlined. In the second section, equations governing the domain growth will be set up for $\frac{d-d_{n}}{L}>\frac{1}{2}$. Focus will be given on the peculiar hydrodynamic behaviour expected in these films. In the third and last section, the exact solution for the evolution of a single hole in the middle of a finite film will be given. 


\section{Molecular model for the domain edges.}

The structure of smectic liquid crystals has been extensively studied. The lowest energy configuration of the domain edge is the one presented in figure 2. Figure $2 a$ is a schematic representation of the distribution of $A$ and $B$ species. It is supported by the following arguments: $\mathrm{AB}$ film has to cover completely the free surface since $\gamma_{\mathrm{B}}<\gamma_{\mathrm{A}}$. Cohesion
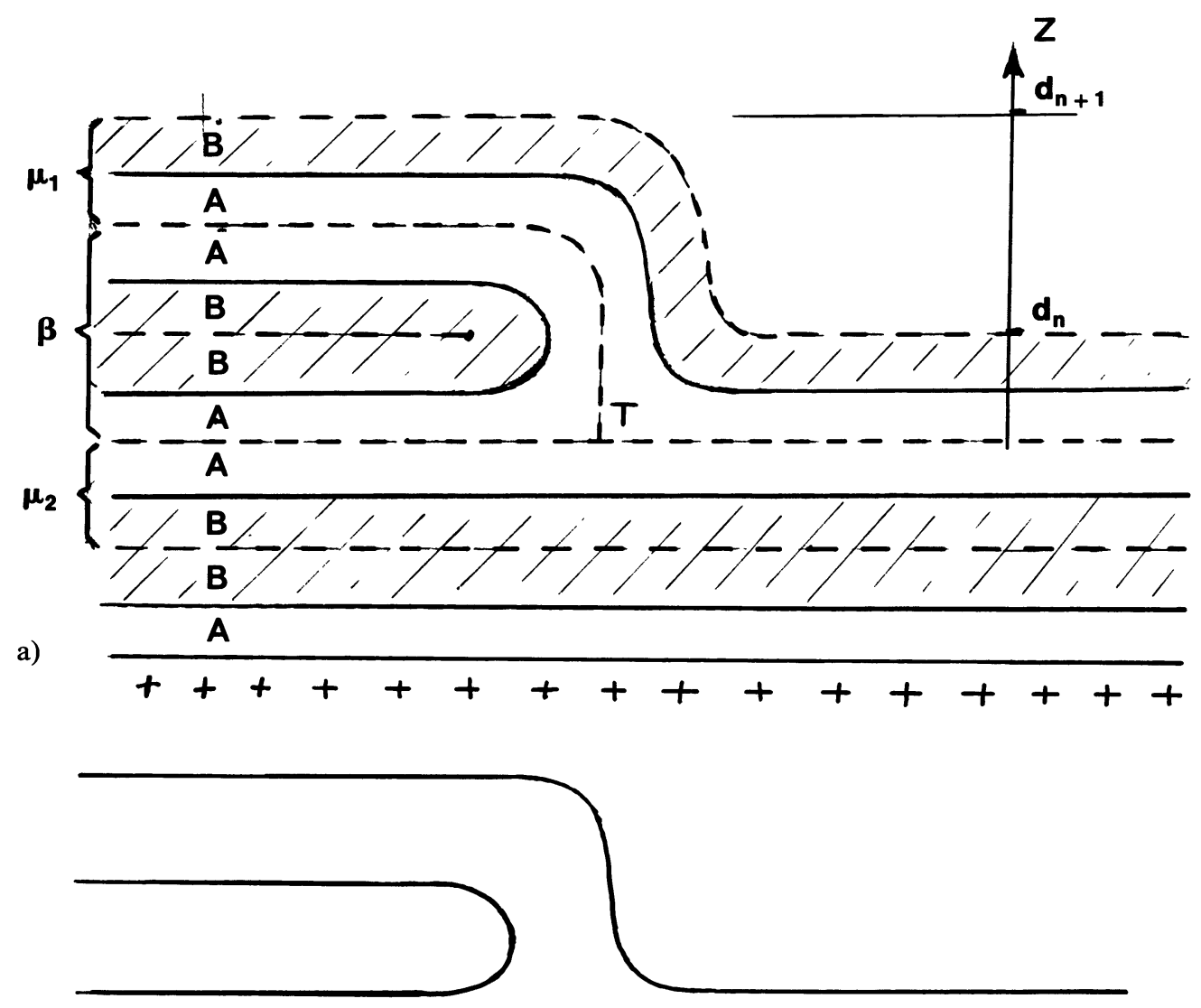

b)

Fig. 2. - a) Assumed domain edge structure. Full lines are representing A-B interface, while dotted lines are for Air-B and fictive A-A or B-B interfaces. Cruces are figurating the solid substrate. The bilayer $\beta$ is trapped in between monolayers $\mu_{1}$ and $\mu_{2}$. The latter are meeting along the triple line $T$ where $\beta$ is disrupted. $\beta$ appears as a monolayer refolded at the edge. Dashed areas are representing B layers. White layers are continuous. Translational invariance along the direction normal to the paper should be imagined. b) Same as figure 2a. A simplified representation has been used, where only A-B interfaces have been drawn. c) Schematic representation of a hole, in perspective, with the same convention than in figure $2 \mathrm{~b}$. The $\mathrm{B}$ part of the bilayer $\beta$ has been underlined by dashed areas. Dotted lines figure some of the hidden parts. d) Same as figure $2 \mathrm{c}$, but in the case of an island. Note that the bilayer $\beta$ (dashed area) is closed. 
c)

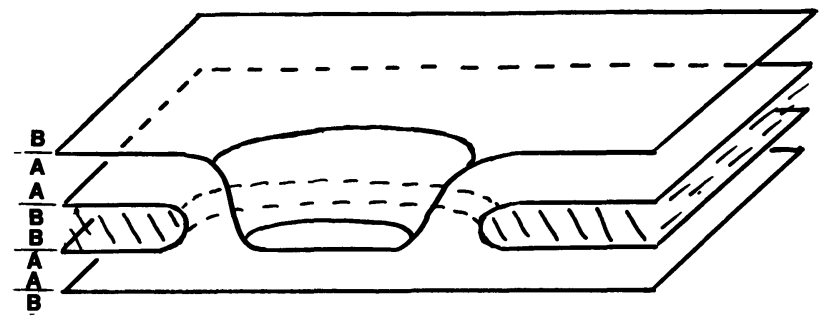

d)

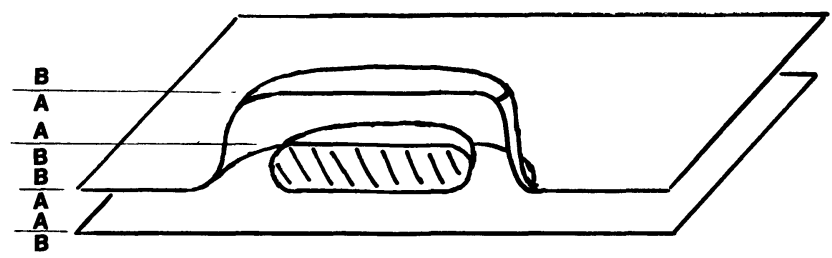

Fig. 2 (continued).

energies of A and B melts are respectively $2 \gamma_{\mathrm{A}}$ and $2 \gamma_{\mathrm{B}}$. The last inequality implies that the B film can be broken more easily than the A film, so that the A film is expected to remain continuous. Finally, the dislocation present at the domain edge could be drawn at different levels into the multilayer sample. However, the number of distorded layers is minimum when the defect is as close as possible from the free surface, and the structure proposed in figure 2 satisfies this last requirement. Also, two disclination lines at different levels would be able to cross each other, and this has never been observed [8]. Note that the domain height is always $L$, as observed on experimental systems [5]. A direct consequence of the structure is that the domain edge width should have dimensions comparable with the macromolecular size, i.e. some hundreds of Angströms. Figure $2 b$ is equivalent to figure $2 a$ but the representation is more compact: only the A-B interfaces have been drawn. Using the same convention, figure $2 c$ and $2 d$ illustrate the topological structure of domains expected for holes and islands, respectively. The difference between the two situations is striking. For holes $2 \mathrm{c}$, the ABBA bilayer between levels $Z=d_{n}-\frac{L}{2}$ and $Z=d_{n+1}-\frac{L}{2}$ is topologically continuous. On the contrary in figure $2 \mathrm{~d}$, the same bilayer, which will be refered to in the following as bilayer $\beta$, is closed and trapped in each island. The consequences are drastic : in the former situation, domains can exchange matter by simple two dimensional flows without changing the topology of domains, while in the latter, domains are prevented to exchange copolymer chains without breaking the defect structure at the edge. Islands are therefore expected to be much more stable than holes, and the growth of islands should be thermally activated.

The next basic remark concerns the pressure in bilayer $\beta$. The average curvature $\left\langle\frac{1}{R}\right\rangle$ of its A-B interface is not uniform. The associated pressure jump when crossing it is of order $2 \gamma_{\mathrm{AB}}\left\langle\frac{1}{R}\right\rangle$ according to Laplace formula. Since $\gamma_{\mathrm{AB}}$ is a constant, the pressure difference experienced when entering the bilayer depends on the path choosen. As a consequence, the pressure in the bilayer is not isotropic. We assume that the pressure tensor $P_{i j}$ is uniaxial with its extraordinary axis $z$ normal to the lamellae. Taking $x$ and $y$ as the plane coordinates, we have $P_{x x}=P_{y y}$ and $P_{z z} \neq P_{x x}$ where the indices are obeying standard notations.

We define the two dimensional pressure $\Pi$ in the bilayer as $\Pi=\frac{L}{2} P_{x x}$ 


\section{The growth mechanism for holes.}

All layers are covering completely the sample except bilayer $\beta$ which is interrupted at the domain boundaries. It is therefore the only location where the flows responsible for the domain growth can take place, at least when permeation, i.e. interlayer exchanges of copolymer chains, can be neglected. It is certainly the case when $\frac{d-d_{1}}{L} \gg \frac{1}{2}$ and we now restrict ourselves to that simplest configuration. Then bilayer $\beta$ is continuous with separate holes in it. Also, very dilute systems will be considered, so that coalescence is ignored. Both A-B interfaces of bilayer $\boldsymbol{\beta}$ are moving together and the entire velocity profile in the bilayer is flat, so that the flow is truely two-dimensional. The bilayer will be treated as an incompressible two-dimensional liquid.

The flow induced motion of the edge is illustrated in figure 3 , where the simplified representation of figure $2 b$ has been used. Bilayer $\beta$ is creeping in between immobile adjacent monolayers $\mu_{1}$ and $\mu_{2}$. Monolayer $\mu_{1}$ is moving upward at the edge of an hole which is decreasing in size. It would move downwards at the edge of a growing hole.

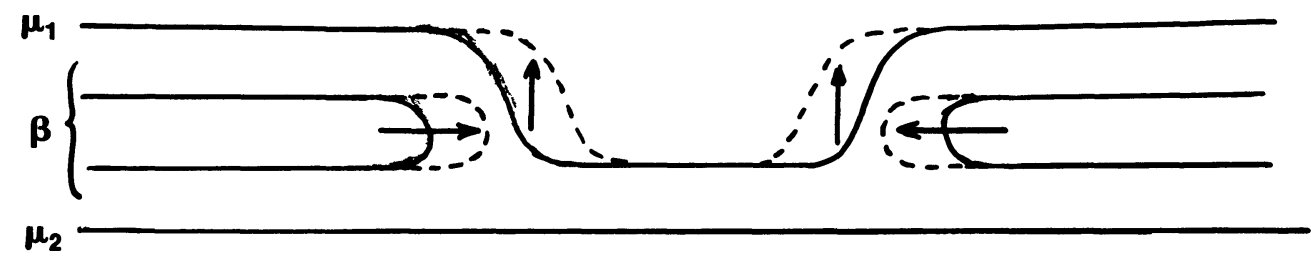

Fig. 3. - Illustrates the mechanism of the edge motion for a hole which is contracting with time. Full lines represent the A-B interfaces at time $t$. Dotted lines are for the same interfaces at $t+$ $\Delta t$. The same convention as in figure $2 \mathrm{~b}$ has been used. The arrows are schematic representations of the layers displacement : $\mu_{1}$ is moving upwards when $\beta$ is advancing lateraly. $\mu_{2}$ is immobile. For a growing hole, invert the arrows and take $\Delta t<0$.

The resistance to the flow has three distinct origins :

a) strong entanglements of the chains in the bilayer induce a high two dimensional viscosity $\eta_{2}$. It resists to relative motions of the chains;

b) chains are also entangled with those of neighbouring monolayers $\mu_{1}$ and $\mu_{2}$. It opposes to absolute motions the chains ;

c) the dislocation at the edge is moving with the bilayer. Polymers of layers $\mu_{1}$ and $\mu_{2}$ are entangled where in contact. Propagation of the dislocation towards this region needs disantenglement of the chains. This is the origin of an additional friction. It ressembles to the resistance to the propagation of a fracture front. Its main characteristic is to introduce a dissymmetry with respect to the direction of the propagation, that is to say between inwards and outwards flows.

It seems that very little has been done on hydrodynamic properties of ordered copolymers, and we have no strong argument to decide which is the dominant friction between mechanisms b) and c). On one hand, intralayer entanglements are much more efficient than interlayer entanglements in lowering chain mobility. On the other hand, chain displacements with respect to immobile layers $\mu_{1}$ and $\mu_{2}$ are orders of magnitude larger than chain displacements relative to their neighbours inside the bilayer. Keeping in mind the latter argument we choose arbitrarily to focus on situations where interlayer frictions are dominant. The bilayer is then considered as an inviscid (non-visquous) two dimensional liquid. 
The current density in the bilayer writes : $\mathbf{J}=\rho L \mathbf{v}$, where $\rho$ is the bulk density and $\mathbf{v}$ the local velocity of the polymer in the bilayer. Since $|\mathbf{v}|$ is very low, the motion of a fluid element is simply described by an exact balance between the local pressure gradient and a solid-like friction term :

$$
\mathbf{J}=-\frac{\nabla \Pi}{\tilde{\eta}}
$$

Note that this Darcy equation for a perfect two-dimensional fluid is formally identical to the kinetic equation of a viscous three-dimensional liquid confined between two solid plates [9]. Were the 2-D viscosity of the copolymer taken into account the analogy would break down.

Assuming 2D incompressibility of the bilayer leads :

$$
\operatorname{div} \cdot \mathbf{v}=\mathbf{0} .
$$

Since $\tilde{\eta}$ is independent of $\mathbf{v},(1)$ and (2) combine in :

$$
\nabla^{2} \Pi=0 .
$$

In order to define completely the 2D-pressure profile $\Pi(x, y)$ in the copolymer bilayer $\beta$, we need to set the appropriate boundary conditions on $\Pi$ at the domain edges. At the interface with domain $i$, the bilayer exhibits two principal radii of curvature, $R_{i}$ and $r_{i}$, respectively into and normal to the $x-y$ plane. $R_{i}$, negative for holes, is also the radius of domain $i ; r_{i}$ is the radius of refolding the A-B interface where $\beta$ is interrupted. Since the copolymer is treated as an incompressible liquid, the thickness of the bilayer is fixed, and $r_{i}$ is a constant along the bilayer frontier. The $2 \mathrm{D}$ pressure $\Pi_{\text {ref }}$ in the top monolayer $\mu_{1}$ is constant with respect to $x$ and $y$. Local mechanical equilibrium between $\beta$ and $\mu_{1}$ requires that the pressure $\Pi_{i}$ in $\beta$ at the vicinity of the edge obeys [10] :

$$
\Pi_{i}=\mathrm{Cte}+\frac{\tau}{4 R_{i}}
$$

where Cte $\simeq \Pi_{\text {ref }}-\frac{2 \gamma_{\mathrm{AB}} L}{r_{i}}$ and $\tau$ is the line tension of the edge defect. We are also interested in the variations of $\Pi$, so that we can replace (4) by :

$$
\Pi_{i}=\frac{\tau}{4 R_{i}}
$$

where $R_{i}<0$.

For a set of $N$ domains $i, i \in[1, N]$, the pressure profile at time $t$ is therefore defined by $N+1$ equations :

$$
\left\{\begin{array}{l}
\nabla^{2} \Pi(t ; x, y)=0 \\
\Pi_{i}(t)=\frac{\tau}{4 R_{i}(t)}
\end{array} .\right.
$$

The resulting quasistationnary profile evolves slowly with time according to the material flow described by (1) in which $\mathbf{J}$ and $\Pi$ are functions of time $t$. The knowledge of $\mathbf{J}$ along the boundary of domain $i$ allows to determine the total flux $\phi_{i}$ of copolymer going out of the domain, and hence the time evolution of its radius $R_{i}(t)$. We thus end out with a self consistent picture able to describe the growth kinetics of an arbitrary distribution of holes. 


\section{The single hole problem.}

We now solve completely the growth problem in the very simple situation where a single hole with radius $\left|R_{1}\right|$ is present at the center of a finite circular film of radius $\left|R_{2}\right|$ at time $t=0$.

We take $R_{1}>0$ and $R_{2}<0$.

The potential $\Pi$ in the bilayer is a function of the distance $r$ from the common center of the two circular edges. The solution of Laplace equation (1) is :

$$
\Pi(r)-\Pi_{\text {ref }}=A \ln r+B
$$

where the constants $A$ and $B$ are to be determined by boundary conditions (2). They presently assume the form:

$$
\left\{\begin{array}{l}
A \ln R_{1}+B=-\frac{\tau}{4 R_{1}} \\
A \ln \left(-R_{2}\right)+B=\frac{\tau}{4 R_{2}}
\end{array} .\right.
$$

It leads :

$$
\begin{aligned}
& A=\frac{\tau}{4 \ln \left(-\frac{R_{2}}{R_{1}}\right)}\left(\frac{1}{R_{1}}-\frac{1}{R_{2}}\right) \\
& B=-\frac{\tau}{4 R_{1}}-A \ln R_{1}
\end{aligned}
$$

or :

$$
\Pi(r)=\frac{\tau}{4} \frac{\left(\frac{1}{R_{1}}-\frac{1}{R_{2}}\right)}{\ln \left(-\frac{R_{2}}{R_{1}}\right)} \ln \left(\frac{r}{R_{1}}\right)-\frac{\tau}{4 R_{1}}
$$

It gives a pressure gradient :

$$
\nabla \Pi=\frac{\tau}{4} \frac{\left(\frac{1}{R_{1}}-\frac{1}{R_{2}}\right)}{\ln \left(-\frac{R_{2}}{R_{1}}\right)} \frac{1}{r} \frac{\mathbf{r}}{r}
$$

From equation (3), the current density at distance $r$ is :

$$
\mathbf{J}(r)=-\frac{\tau}{4 \tilde{\eta}} \frac{\left(\frac{1}{R_{1}}-\frac{1}{R_{2}}\right)}{\ln \left(-\frac{R_{2}}{R_{1}}\right)} \frac{1}{r} \frac{\mathbf{r}}{r}
$$

Setting $\mathbf{J}(r)=J(r) \frac{\mathbf{r}}{r}$, the mass flux $\phi(r)$ through a circular line at distance $r$ is

$$
\phi(r)=2 \pi r J(r)
$$


From mass conservation, we also have :

$$
\phi(r)=\rho L \frac{\mathrm{d}}{\mathrm{d} t}\left(\pi R_{1}^{2}\right) .
$$

Identifying (12) and (13) leads :

$$
R_{1} \frac{\mathrm{d} R_{1}}{\mathrm{~d} t}=-\frac{\tau}{4 \tilde{\eta} \rho L} \frac{\left(\frac{1}{R_{1}}-\frac{1}{R_{2}}\right)}{\ln \left(-\frac{R_{2}}{R_{1}}\right)}
$$

The conservation of mass at the boundaries also gives

$$
R_{1} \frac{\mathrm{d} R_{1}}{\mathrm{~d} t}=+R_{2} \frac{\mathrm{d} R_{2}}{\mathrm{~d} t}
$$

Equations (14) and (15) are governing the time evolution of the two circular edges. We solve equation (14) when $R_{1} \ll R_{2}$. It becomes :

$$
R_{1}^{2} \frac{\mathrm{d} R_{1}}{\mathrm{~d} t}=-\frac{\tau}{4 \tilde{\eta} \rho L \ln \left(-R_{2}\right)} .
$$

From (15), we have $\frac{\mathrm{d}\left(-R_{2}\right)}{\mathrm{d} t} \ll \frac{\mathrm{d} R_{1}}{\mathrm{~d} t}$ and $\ln \left(-R_{2}\right)$ can be treated as a constant. Hence :

$$
R_{1}^{3}(t)=\frac{3}{4} \frac{\tau}{\tilde{\eta} \rho L \ln \left(-R_{2}\right)}\left(t_{\max }-t\right) .
$$

The time $t_{\max }$ where hole 1 disappears is a function of its radius $R_{0}$ at $t=0$ :

$$
t_{\max }=\frac{4 \tilde{\eta} \rho L R_{0}^{3} \ln \left(-R_{2}\right)}{3 \tau} .
$$

This result and equations of part 2 are suggestive of a close analogy between the growth mechanism considered herein and the last stage kinetics of first order transitions [7].

Whether the degeneracy of the copolymer film into domains of different thicknesses can be considered as a true first order two-dimensional phase transition or not will be the subject of forthcoming developments.

It is important at this point to underline two major features of our copolymer systems : first, broadening of interfaces with time is not supposed to occur; secondly, matter exchange between domains is expected to be convective in nature (i.e. pressure driven) rather than diffusive (driven by concentration gradients) as encountered in Ostwald ripening.

\section{Acknowledgments.}

We are indebted to P. G. de Gennes and D. Andelman for illuminating comments. We also thank C. M. Knobler and J. Friedel for stimulating discussions. This work has been supported by the C.N.R.S. 


\section{References}

[1] Skoulios A., Adv. Liq. Cryst. 1 (1975) 169 ;

LEIBLER L., Macromolecules 13 (1980) 1602 ;

Ed. Aggawal S., Block copolymers (Plenum Press) 1970.

[2] Skoulios A., Block and Graft Copolymers (Syracuse University Press) 1973.

[3] De Gennes P. G., Suppl. to Solid State Phys. (Ehrenreich, Academic Press) 1977 ; DE Gennes P. G., Macromolecules 13 (1980) 1069.

[4] Coulon G., Russell T. P., Deline V. R., Green P. F., Macromolecules 22 (1989) 2581.

[5] Coulon G., Ausserré D. and Russell T. P., J. Phys. France 51 (1990) 777.

[6] Gunton J. D., SAn Miguel M. and Sahni P., Phase Transitions and Critical Phenomena, Eds. C. Domb and J. L. Debowitz (Academic, London) 8 (1983) 267.

[7] Voorhees P. W., J. Stat. Phys. 38 (1985) 231.

[8] Bouligand R., private communication.

[9] LAMB H., Hydrodynamics (Dover, New York) 1945, chapter XI.

[10] KaWASAKi K. and OHTA T., Physica 118A (1983) 175. 